
março 2.000

\title{
Avaliação de cultivares de cebola em Petrolina-PE.
}

\author{
Nivaldo Duarte Costa; Geraldo Milanez de Resende; Rita de Cássia Souza Dias \\ Embrapa Semi-Árido, C. Postal 23, 56.300-000 Petrolina-PE.
}

\section{RESUMO}

Objetivando-se indicar cultivares de cebola mais produtivas e de melhor qualidade de bulbos para a região semi-árida, conduziuse um ensaio no Projeto Bebedouro, Petrolina-PE, de abril a agosto de 1997. O delineamento experimental foi de blocos ao acaso com quatro repetições e as seguintes cultivares: Granex-429, Texas GranoPRR, Brownsville, Houston, Texas Grano-502, Texas Grano-438, Conquista, Composto IPA-6, Belém IPA-9, Franciscana IPA-10, Valeouro IPA-11, Alfa Tropical (CNPH-6179), CNPH-5898, CNPH6074, CNPH-6040, CNPH-6067, Bola P. EMPASC, XP-1, XP-2 e Crioula Mercosul. A produtividade de bulbos comerciais variou de 21,41 a $61,78 \mathrm{t} / \mathrm{ha}$, destacando-se as cultivares Texas Grano-PRR $(61,78 \mathrm{t} / \mathrm{ha})$, Granex-429 (58,28 t/ha), Texas Grano-438 (56,97 t/ ha), Brownsville (55,38 t/ha), Texas Grano-502 (53,97t/ha) e Houston $(53,35 \mathrm{t} / \mathrm{ha})$, que não mostraram diferenças significativas entre si.

Palavras chave: Allium cepa, adaptação, produtividade, classificação de bulbos.

\begin{abstract}
Evaluation of onion cultivars at Petrolina, Pernambuco State, Brazil.
\end{abstract}

With the objective of identifying high productive and better quality onion cultivars for the tropical semi-arid region of Brazil, field trials were conducted at Petrolina, Pernambuco State, Brazil, from February until July of 1996.The experimental design was a randomized complete blocks design with four repetitions and twenty treatments (Cultivars Granex-429, Texas Grano-PRR, Brownsville, Houston, Texas Grano-502, Texas Grano-438, Conquista, Composto IPA-6, Belém IPA-9, Franciscana IPA-10, Valeouro IPA-11, Alfa Tropical (CNPH-6179), CNPH-5898, CNPH-6074, CNPH-6040, CNPH-6067, Bola P. EMPASC, XP-1, XP-2 and Crioula Mercosul). Production of commercial bulbs ranged forms 21.41 to 61.78 ton/ ha. Texas Grano-PRR had the highest yield (61.78 ton/ha), followed by Granex-429 (58.28 ton/ha), Texas Grano-438 (56.97 ton/ha), Brownsville (55.38 ton/ha), Texas Grano-502 (53.97 ton/ha) and Houston (53.35 ton/ha).

Keywords: Allium cepa, adaptation of cultivars, yield, bulb classification.

(Aceito para publicação em 07 de fevereiro de 2.000)

A produção mundial de cebola (Allium cepa $\mathrm{L}$.) nos últimos anos (1995/96/97) esteve entre 37,38 e 38,49 milhões de t/ano, proveniente de uma área de cultivo de 2,2 a 2,3 milhões de hectares/ano, com uma produtividade média de 16,7 t/ha (FAO,1998). No Brasil, a cebola destaca-se ao lado da batata e do tomate como a cultura olerácea economicamente mais importante, tanto pelo volume produzido, em torno de 900 mil t/ano, como pela renda gerada. A sua produção ocorre nas regiões Sul (Paraná, Santa Catarina e Rio Grande do Sul), contribuindo com $37,7 \%$ da produção nacional, Sudeste (São Paulo e Minas Gerais), com 35,3\% e Nordeste (Pernambuco e Bahia), com 27\%. A produtividade média nacional se situa em $12,49 \mathrm{t} / \mathrm{ha}$, sendo que nos estados de Pernambuco e Bahia, maiores produtores do Nordeste, a produtividade média é de 14,89 e 14,68 t/ha (ANUÁRIO, 1996).

No contexto do Mercosul, destacamse apenas as produções do Brasil, com ofertas equivalentes às suas necessida- des de consumo, e da Argentina, cujo volume de produção tem gerado expressivos excedentes exportáveis. Esses dois países respondem em sua quase totalidade pelo abastecimento do referido mercado, sendo as produções do Uruguai e Paraguai praticamente marginais (cerca de 3,5\%), não influindo no perfil do mercado. (Boeing, 1995).

A globalização da economia mundial e a formação do Mercosul interferiram significativamente no mercado de hortaliças no Brasil. As tendências das produções na Argentina e no Brasil evidenciam um mercado competitivo do qual continuarão participando aqueles países que tiverem maiores vantagens comparativas e fizerem inversões nos setores produtivos. Portanto, o momento por que passa a cebolicultura é crucial e deve apresentar definições. Somente continuará no mercado o produtor que se tecnificar, obter produto de qualidade e se adaptar a essas mudanças no mercado (Ferreira,1997). Hoje, a cebola está sendo cultivada em regiões distintas, dentro de uma grande amplitude geográfica, estendendo-se do Equador até regiões mais próximas aos círculos polares.

As pesquisas têm demonstrado que as melhores cultivares são aquelas obtidas na própria região de produção, porque cada uma requer condições especiais de fotoperíodo e temperatura para a obtenção das características qualitativas desejáveis, altos rendimentos e boa conservação no armazenamento (Jones, 1963).

A cultivar Texas Grano é uma das mais adaptadas às condições do Centro Sul do País (Filgueira,1982). Goto \& Costa (1979), avaliando cultivares, concluíram ser a "Texas Grano-502" a mais produtiva em duas localidades onde foram avaliadas, com respectivamente, 80,0 e 96,8 t/ha.

Avaliando cultivares de cebola de ciclo precoce, Gandim et al. (1989), verificaram produtividades variando de 17,47 a $32,32 \mathrm{t} / \mathrm{ha}$, destacando-se as cultivares EMPASC 352- Bola precoce (32,32 t/ha), IPA-1 (29,42 t/ha), Baia Periforme $(28,88 \mathrm{t} / \mathrm{ha})$, IPA-2 $(28,25 \mathrm{t} /$ 
Tabela 1. Produtividade total e comercial de cultivares de cebola no Vale São Francisco. Embrapa Semi-Árido, Petrolina (PE), $1997^{1}$.

\begin{tabular}{|c|c|c|c|c|}
\hline \multirow{2}{*}{ Cultivares } & \multicolumn{4}{|c|}{ Produtividade (t/ha) } \\
\hline & \multicolumn{2}{|c|}{ Total } & \multicolumn{2}{|c|}{ Comercial } \\
\hline Texas Grano-PRR & \multicolumn{2}{|c|}{$62,71 \mathrm{a}$} & \multicolumn{2}{|c|}{$61,78 \mathrm{a}$} \\
\hline Granex-429 & \multicolumn{2}{|c|}{$58,49 a b$} & \multicolumn{2}{|c|}{$58,28 a b$} \\
\hline Texas Grano-438 & \multicolumn{2}{|c|}{$57,79 a b$} & \multicolumn{2}{|c|}{$56,97 a b$} \\
\hline Brownsville & \multicolumn{2}{|c|}{$56,02 a b$} & \multicolumn{2}{|c|}{$55,38 a b$} \\
\hline Texas Grano-502 & \multicolumn{2}{|c|}{$54,20 a b$} & \multicolumn{2}{|c|}{$53,97 a b$} \\
\hline Houston & \multicolumn{2}{|c|}{$53,92 a b$} & \multicolumn{2}{|c|}{$53,35 a b$} \\
\hline Alfa Tropical (CNPH-6179) & 49,86 & bc & 48,96 & bc \\
\hline CNPH-6067 & 43,82 & $\mathrm{~cd}$ & 43,34 & $\mathrm{~cd}$ \\
\hline Franciscana IPA-10 (roxa) & 42,99 & $\mathrm{~cd}$ & 42,68 & $c d$ \\
\hline Valeouro IPA-11 & 40,25 & cde & 39,54 & cde \\
\hline CNPH-6074 & 39,50 & de & 38,86 & cde \\
\hline CNPH-5898 & 36,26 & de & 35,49 & de \\
\hline Composto IPA-6 & 35,62 & de & 34,73 & de \\
\hline$X P-1$ & 35,37 & de & 33,77 & de \\
\hline Belém IPA-9 & 35,22 & de & 33,76 & de \\
\hline Bola P. EMPASC & 33,79 & def & 32,40 & ef \\
\hline CNPH-6040 & 30,93 & efg & 30,36 & ef \\
\hline Conquista & 29,89 & efg & 29,40 & 1 \\
\hline Crioula Mercosul & 24,70 & $\mathrm{fg}$ & 22,31 & 1 \\
\hline$X P-2$ & 23,89 & $\mathrm{fg}$ & 21,41 & 1 \\
\hline C.V. (\%) & 15,42 & & 16,56 & \\
\hline
\end{tabular}

${ }^{1}$ Médias seguidas pela mesma letra nas colunas, não diferem entre si, pelo teste de Duncan, ao nível de $5 \%$ de probabilidade.

ha) e IPA-6 (27,65 t/ha). Estudando a adaptação de cultivares de cebola, Churata-Masca \& Santos (1983), observaram que a "Granex Yellow " foi a mais produtiva (77,3 t/ha), seguida pelas cultivares Excel, Granex-33 e Granex-429 (entre 64,5 e 61,9 t/ha) e "IPA-2" (55,2 t/ha) e "Ag.-59" (50,7 t/ha). Candeia, et al, (1985), verificaram uma produtividade de 30,3 t/ha para a cultivar Brownsville, que foi a mais produtiva dentre as avaliadas. A maior produtividade de bulbos comerciais foi apresentada pelas cultivares Granex-33 e Granex-429 com 33,07 e 32,19 t/ha, respectivamente (Caraballo et al.,1990).

Avaliando 29 cultivares de cebola, Madisa (1994) constatou ser "Equanex" a mais produtiva $(36,28 \mathrm{t} / \mathrm{ha})$, tendo a cultivar Granex alcançado 26,83 t/ha e a produtividade média das cultivares se situado em 23,29 t/ha. Murakami et al. (1995), estudando o comportamento de cinco genótipos e três cultivares, ob- servaram que a cultivar Régia foi a mais produtiva (90,3 t/ha), seguida pela cultivar São Paulo (78,7 t/ha).

Dada a importância desta hortaliça para a região do vale do São Francisco, objetivou-se identificar cultivares adaptadas, que apresentem alto potencial para produção e qualidade de bulbos.

\section{MATERIAL E MÉTODOS}

O experimento foi instalado no Campo Experimental de Bebedouro, da Embrapa Semi-Árido, em Petrolina (PE), com altitude de $365,5 \mathrm{~m}$ e latitude de $9^{\circ} 24^{\prime}$ Sul, no período de abril a agosto de 1997, em Latossolo Vermelho Amarelo com as seguintes características: $\mathrm{pH}\left(\mathrm{H}_{2} \mathrm{O}\right)=7,0 ; \mathrm{Ca}^{2+}=2,2 \mathrm{cmol}_{\mathrm{c}} /$ $\mathrm{dm}^{3} ; \mathrm{Mg}^{2+}=0,8 \mathrm{cmol} / \mathrm{dm}^{3} ; \mathrm{Na}^{+}=0,05$ $\mathrm{cmol} / \mathrm{dm}^{3} ; \mathrm{K}^{+}=0,32^{\mathrm{c}} \mathrm{cmol} / \mathrm{dm}^{3} ; \mathrm{Al}^{3}=$ $0,05 \mathrm{cmol}_{\mathrm{c}} / \mathrm{dm}^{3}$; e P/Mehlich $=23,88$ $\mathrm{mg} / \mathrm{L}$, determinadas segundo metodologia da Embrapa (1979).
O delineamento experimental foi blocos ao acaso com quatro repetições e os tratamentos, constituídos das seguintes cultivares: Granex-429, Texas Grano-PRR, Brownsville, Houston, Texas Grano-502, Texas Grano-438, Conquista, Composto IPA-6, Belém IPA-9, Franciscana IPA-10, Valeouro IPA-11, Alfa Tropical (CNPH-6179), CNPH-5898, CNPH-6074, CNPH6040, CNPH-6067, Bola Precoce EMPASC, XP-1, XP-2 e Crioula Mercosul. Utilizaram-se parcelas de 7,2 $\mathrm{m}^{2}$ de área útil, com 300 plantas por parcela e bordaduras laterais, no espaçamento de $0,15 \times 0,10 \mathrm{~m}$.

A adubação básica constou de $30 \mathrm{Kg} /$ ha de $\mathrm{N} ; 120 \mathrm{~kg} / \mathrm{ha}$ de $\mathrm{P}_{2} \mathrm{O}_{5}$ e $60 \mathrm{~kg} / \mathrm{ha}$ de $\mathrm{K}_{2} \mathrm{O}$, de acordo com as recomendações baseadas na análise do solo. Foram aplicados em cobertura $90 \mathrm{~kg} / \mathrm{ha}$ de $\mathrm{N}$ (uréia) e $60 \mathrm{~kg} / \mathrm{ha}$ de $\mathrm{K} \mathrm{O}$ (cloreto de potássio) parcelados aos 20 e 30 dias após o transplante. Os tratos culturais e controle fitossanitários foram realizados de acordo com as recomendações regionais para a cultura da cebola. $\mathrm{O}$ transplante das mudas foi realizado aos 30 dias após a semeadura e as irrigações, quando necessárias, foram realizadas em sulcos.

A colheita foi efetuada quando a maioria das plantas encontravam-se tombadas, aos 120 dias após a semeadura para as cultivares Granex-429, Texas Grano PRR, Texas Grano 438, Conquista, Alfa Tropical (CNPH-6179), CNPH-5898, CNPH-6074, CNPH6040, Franciscana IPA-10 e Bola Precoce EMPASC, e aos 127 dias para as cultivares Brownsville, Composto IPA6, Belém IPA-9, Valeouro IPA-11 e CNPH-6067 e aos 150 dias para as cultivares XP-1, XP-2 e Crioula Mercosul.

As plantas colhidas foram submetidas ao processo de cura, ficando por seis dias expostas ao sol e dois dias à sombra, efetuando-se, em seguida, o corte da parte aérea. Avaliaram-se as seguintes características: produtividade total $(\mathrm{t} /$ ha) e comercial (t/ha), sendo considerados comerciáveis os bulbos entre $35 \mathrm{e}$ $90 \mathrm{~mm}$ de diâmetro. Os bulbos foram classificados de acordo com o maior diâmetro transversal em quatro classes comerciais, segundo Brasil (1995), onde $2=35$ a $50 \mathrm{~mm}, 3=50$ a $70 \mathrm{~mm}, 4=70$ a $90 \mathrm{~mm}$ e $5=$ bulbos com diâmetro transversal maior que $90 \mathrm{~mm}$; sendo que 
Tabela 2. Percentagem em peso de bulbos comerciais de cultivares de cebola, segundo o maior diâmetro transversal em classes e refugos. Embrapa Semi-Àrido, Petrolina (PE), 1997.

\begin{tabular}{lrrrrr}
\hline \multirow{2}{*}{ Cultivares } & \multicolumn{5}{c}{ Classes $^{*}(\%)$} \\
\cline { 2 - 5 } & $\mathbf{5}$ & $\mathbf{4}$ & $\mathbf{3}$ & $\mathbf{2}$ & $\mathbf{1}^{\text {** }}$ \\
\hline Granex-429 & 49,35 & 38,59 & 10,94 & 0,77 & 0,35 \\
T. Grano-PRR & 51,80 & 32,96 & 12,67 & 2,22 & 0,35 \\
Brownsville & 50,20 & 32,40 & 14,36 & 1,90 & 1,14 \\
Houston & 44,10 & 38,50 & 14,30 & 2,00 & 1,10 \\
T. Grano-502 & 34,00 & 45,00 & 18,60 & 2,00 & 0,40 \\
T. Grano-438 & 51,40 & 33,00 & 12,10 & 2,10 & 1,40 \\
Conquista & 0,80 & 31,20 & 58,00 & 9,00 & 1,00 \\
Composto IPA-6 & 12,27 & 44,72 & 34,50 & 6,04 & 2,47 \\
Belém IPA-9 & 12,02 & 40,50 & 36,85 & 6,50 & 4,13 \\
Valeouro IPA-11 & 6,46 & 44,50 & 42,84 & 4,44 & 1,76 \\
Alfa Trop. (CNPH-6179) & 31,75 & 46,00 & 19,13 & 1,32 & 1,80 \\
CNPH-5898 & 11,00 & 44,60 & 35,80 & 6,50 & 2,10 \\
CNPH-6074 & 17,04 & 46,37 & 30,40 & 4,57 & 1,62 \\
CNPH-6040 & 4,03 & 42,73 & 41,39 & 10,00 & 1,85 \\
CNPH-6067 & 18,84 & 50,40 & 26,10 & 3,50 & 1,16 \\
Franciscana IPA-10 (roxa) & 11,86 & 51,50 & 33,90 & 2,00 & 0,74 \\
B. P. Empasc & 5,95 & 40,76 & 42,50 & 7,65 & 3,14 \\
XP-1 & 11,00 & 44,25 & 34,70 & 5,35 & 4,70 \\
XP-2 & 3,77 & 31,66 & 40,37 & 10,60 & 13,60 \\
C. Mercosul & - & 37,35 & 43,25 & 9,70 & 9,70
\end{tabular}

*Classe 2: 35 a 50mm; Classe 3: 50 a $70 \mathrm{~mm}$; classe 4: 70 a $90 \mathrm{~mm}$ e classe 5: bulbos maiores que $90 \mathrm{~mm}$ de diâmetro transversal.

** Bulbos de baixo valor comercial

os bulbos menores (diâmetro transversal inferior a $35 \mathrm{~mm}$ ) foram computados apenas na produtividade total.

Posteriormente, foi feita a análise de variância das características avaliadas, aplicando-se o teste de Duncan no nível de $5 \%$ de probabilidade para comparação das médias, segundo metodologia descrita por Gomes (1987).

\section{RESULTADOS E DISCUSSÃO}

A colheita foi efetuada quando a maioria das plantas encontravam-se tombadas, sendo iniciada aos 120 dias após a semeadura. Pela Tabela 1, observa-se que a produtividade de bulbos comerciais variou de 21,41 a $61,78 \mathrm{t} /$ ha, destacando-se as cultivares Texas Grano-PRR, Granex-429, Texas Grano438, Brownsville, Texas Grano-502 e Houston, que não mostraram diferenças significativas entre si. Estas cultivares apresentaram incrementos na produtivi- dade da ordem de 285,5 a $386,4 \%$ superiores à média nacional (12,7 t/ha); estando ainda acima da produtividade média mundial de 16,7 t/ha e dos resultados encontrados por Gandim et al. (1989), Candeia et al. (1985) e Madisa (1994) que variaram de 17,47 a 36.28 t/ ha. Entretanto foram inferiores às produtividades relatadas por Churata-Masca \& Santos (1995) e Murakami et al. (1995), que relataram produtividades entre 77,3 e 90,3 t/ha. Os mais baixos rendimentos foram observados para as cultivares Bola Precoce EMPASC, CNPH-6040, Conquista, Crioula Mercosul e XP-2, que não mostraram diferenças estatísticas entre si. A boa performance produtiva das cultivares Texas Grano-PRR e Texas Grano-438, são evidenciadas por Goto \& Costa (1979) e Filgueira (1982), que relatam serem estas as mais adaptadas e produtivas. Assim como também em relação a cultivar Granex-429, que Caraballo et al. (1990), informam boa produtividade comercial de bulbos (32,19 t/ha), no entanto, inferior a alcançada no presente trabalho.

A Tabela 2, apresenta a percentagem do peso de bulbos comerciais de cebola em classes, segundo o maior diâmetro transversal. As cultivares Conquista, Valeouro IPA -11, Franciscana IPA-10, CNPH-6040, Bola P. EMPASC, CNPH5898 e Crioula, sobressaíram-se das demais por apresentarem bulbos comerciais acima de $80 \%$ nas classes 3 (50 a $70 \mathrm{~mm}$ de diâmetro transversal) e da classe 4 (70 a 90mm de diâmetro transversal), que são os de maior preferência do mercado consumidor nacional. A cultivar XP-2 apresentou a maior quantidade de bulbos tipo 1 (chupeta), assim como as cultivares Texas Grano-PRR, Texas Grano-438, Brownsville, Granex-429 e Houston apresentaram $44 \%$ de bulbos comerciais da classe 5 (acima de $90 \mathrm{~mm}$ de diâmetro transver- 
sal), que são bulbos grandes de baixo valor comercial, conforme relatam Silva et al.(1991) da preferência do consumidor nacional por bulbos com 80 a $100 \mathrm{~g}$ e diâmetro transversal de 40 a $80 \mathrm{~mm}$.

Os resultados obtidos em termos de produtividade e qualidade de bulbos evidenciam que poderiam ser utilizadas pelos produtores, sob irrigação, as cultivares: Franciscana IPA-10, Valeouro IPA11, Alfa Tropical (CNPH-6179) e CNPH6067. Assim como consolida a cultivar Texas Grano-502 como adaptada às condições do vale do São Francisco.

As cultivares Granex-429, Brownsville, Texas Grano-PRR, Houston e Texas Grano- 438, pela sua performance produtiva, necessitam de maiores estudos quanto a níveis de adubação e densidades de plantio, que promovam menor diâmetro do bulbo, adequando-as à preferência do mercado consumidor.

\section{BIBLIOGRAFIA CITADA}

ANUÁRIO ESTATISTICO DO BRASIL. Rio de janeiro: IBGE, v. 56, 1996.
BOEING, G Cebola. Florianópolis: Instituto CEPA/SC, 85 p. 1995

BRASIL. Ministério da Agricultura, Abastecimento e Reforma Agrária, Portaria n. 529 de 18 ago. Diário Oficial (República Federativa do Brasil), Brasília, 1 set.,Seção1, p. 13513. 1995.

CANDEIA, J.A.; MENEZES, J.T. de; MENEZES, D.; WANDERLEY, L.J. da G. Avaliação de cultivares de cebola no Submédio São Francisco. In: CONGRESSO BRASILEIRO DE OLERICULTURA, 25, Blumenau, SC. Resumos... Sociedade de Olericultura do Brasil, p. 65. 1985.

CHURATA-MASCA, M.G.C.; SANTOS, M A.P. dos. Competição de cultivares de cebola. In: CONGRESSO BRASILEIRO DE OLERICULTURA, 23, Rio de Janeiro, RJ. Resumos... SOB. p. 36. 1983.

CARABALLO, E.; FORNARIS, G.J.; GUADALUPE, R.; HERNADEZ, E.R. de. Performance, sizing and total solids of nine onion( Allium cepa L.) cultivars. Journal of Agriculture of the Puerto Rico, v. 74, n. 1, p. 21-27, 1990

EMBRAPA. Serviço Nacional de Levantamento e Conservação de solos. Manual de métodos de análise de solo. Rio de Janeiro: EMBRAPASNLCS,1979. (Não paginado).

FAO QUATERLY BULLETIN OF STATISTICS. Rome: FAO, v. 11, n. 112, 1998.

FERREIRA, M.D. Chegou o tempo da segmentação. Agrianual, São Paulo, p. 190195,1997
FILGUEIRA, F.A.R. Manual de olericultura: cultura e comercialização das hortaliças. São Paulo: Agronômica Ceres, v. 2, 1982. 357 p.

GANDIN, C.L.; TORRES, L.; GUIMARÃES, D.R.; THOMAZELLI, L.F.; DITRICH, R.C. Competição de cultivares de cebola. Agropecuária Catarinense, v. 2, n. 2, p. 5254. 1989.

GOMES, F.P. Curso de estatística experimental. 12 ed., 467 p. 1987.

GOTO, R.; COSTA, J.A. Comportamento de cultivares de cebola sob irrigação, no municipio de Irecê-BA. Salvador: EPABA, 1979. 14 p. (EPABA. Comunicado técnico, 43).

JONES, H.A. ; MAN, L.K. Onion and their allies New York, Interscience, 1963. 283 p.

MADISA, M.G. Onion cultivars trials for yield and storage in Botswana, 1992-1993. Onion Newsletter for the Tropics. n. 6, p. 38-44, 1994.

MURAKAMI, J.; ARAÚJO, M. de T.; CHURATA-MASCA, M.G.C. Avaliação de genótipos selecionados de cebola. Horticultura Brasileira, Brasília, v. 13, n. 1, p. 95.1995.

SILVA, E.; TEIXEIRA, L.A.J.; AMADO, T.J.C The increase in onion production in Santa Catarina, State, South, Brazil. Onion Newsletter for the Tropics. n. 3, p. 7-9, 1991. 